To be published in Optics Letters:

Title: Vertical-cavity surface-emitting laser with liquid crystal external cavity Authors: Yi Xie, Jeroen Beeckman, Krassimir Panajotov, and Kristiaan Neyts Accepted: 4 October 2014

Posted: 16 October 2014

Doc. ID: 221862 


\title{
Vertical-cavity surface-emitting laser with liquid crystal external cavity
}

\author{
Yi Xie, ${ }^{1,2, *}$ Jeroen Beeckman, ${ }^{1,2}$ Krassimir Panajotov ${ }^{3}$ and Kristiaan Neyts, ${ }^{1,2}$ \\ 1 Department of Electronics and Information Systems, Ghent University, Ghent 9000, Belgium \\ 2 Center for Nano and Biophotonics, Ghent University, Ghent 9000, Belgium \\ 3 Department of Applied Physics and Photonics, Vrije Universiteit Brussels, Brussels 1000, Belgium \\ *Corresponding author: yi.xie@ugent.be \\ Received Month X, XXXX; revised Month X, XXXX; accepted Month X, \\ XXXX; posted Month X, XXXX (Doc. ID XXXXX); published Month X, XXXX
}

\begin{abstract}
A tuneable external cavity consisting of a thin layer of nematic liquid crystal (LC) and a dielectric reflector is placed on the top of a vertical-cavity surface-emitting laser (VCSEL). By changing the voltage across the LC layer, the optical path length of the external cavity can be tuned. As a result, the emitting properties of the LC-VCSEL, including polarization state and emission wavelength can be controlled by the voltage applied over the LC layer. Stable polarization switching with high contrast is obtained by voltage driving. This device can be integrated in applications which require electrically tuneable VCSEL emission. (C2014 Optical Society of America
\end{abstract}

OCIS codes: (140.7260) Vertical cavity surface emitting lasers; (230.3720) Liquid-crystal devices.

Controlling the emission properties, such as polarization state and emission wavelength, of verticalcavity surface-emitting lasers (VCSELs) is interesting for many applications, ranging from optical sensing to optical communication. In current optical communication systems, the optical signals are modulated with frequencies of several tens of $\mathrm{GHz}$, many applications such as packet switching or protective switching do not need this speed. Different ways to achieve this have been intensively studied. Theoretical and experimental results on controlling the transverse modes, polarization states and longitudinal modes of VCSELs have been reported in the past [1-11]. From the theoretical analysis of the operation of a VCSEL with an external cavity [3, 4], the polarization mode can be switched by either changing the strength or the delay of the optical feedback (OF) or using a current-dependent thermal effect. Polarization switching is realized by current injection [5], phase change in the external cavity [6], temperature [7], frequency modulation [8], or optical injection [9, 10]. Liquid crystal (LC) is used in VCSELs to obtain a tunable lasing wavelength [11]. Drawbacks of these approaches are the limited contrast of polarization switching in [5], the mechanical control to change the external cavity length in [6] and the optical pumping in [9-11] which severely limits the applicability.

In our previous work, we have demonstrated the integration of an electrically driven VCSEL chip into a liquid crystal cell formed by two glass plates [12]. Based on this device design, the polarization of the VCSEL emission can be modulated by adding voltage-tunable birefringent optical materials [12]. In this device, LC is used as a polarization modulator which does not provide optical feedback to the VCSEL. This means that the switching time is directly related to the switching time of the liquid crystal, which is typically several tens of milliseconds. In the second generation of this device, more functions are realized by adding optical feedback introduced by chiral nematic LC [13, 14]. The chiral nematic LC is essentially a periodic structure with a reflection band which acts as a temperature-tunable top mirror of the VCSEL cavity. In the current work, we demonstrate an electrically tunable external cavity. The device consists of a thin dielectric reflector deposited on the top glass substrate and a thin nematic LC layer forming the external optical cavity. The optical length of this external cavity is tuned by the voltage applied across the LC layer. We demonstrate that the polarization state, the threshold current and the emission wavelength can be controlled by changing the voltage applied across the LC layer. Due to the optical feedback of the external LC cavity, the switching time of the polarization state of the VCSEL is now largely determined by the laser dynamics of the cavity, which in theory is in the nanosecond range. In this work we observe switching times in the order of hundred microseconds, which is much faster than the LC switching time.

A single mode VCSEL chip (ULM850-SingleMode_OMv14) emitting at $850 \mathrm{~nm}$ wavelength is incorporated between two glass plates according to the procedure described in [12]. The dimensions of the VCSEL chip are $250 \mu \mathrm{m} \times 250 \mu \mathrm{m} \times 150 \mu \mathrm{m}$. The side view of the device is shown in Fig. 1A. The top periodic $\mathrm{SiO}_{2} / \mathrm{Ta}_{2} \mathrm{O}_{5}$ bi-layers have thicknesses of $120 \mathrm{~nm} / 84 \mathrm{~nm}$ which results in the spectral transmission diagram of Fig. 1B. The cathode and anode of the VCSEL are electrically connected to the bottom ITO and the top chromium electrode respectively, through micro-sized gold coated silica balls embedded in the UV cured optical glue. The diameter of these balls is $10 \mu \mathrm{m} \pm 0.1 \mu \mathrm{m}$. The physical length of the cavity $(d$ shown in Fig. 1A) between the VCSEL emitter and the external reflector is determined by the diameter of these spacers. The alignment of the LC molecules in the external cavity is achieved by a photo-alignment layer, and its planar alignment direction is chosen to be the same as the original linear polarization of the VCSEL emission (polarization $\mathrm{P}_{\mathrm{x}}$ as along the $\mathrm{x}$ axis). The optical power and emission wavelength are measured with an optical setup consisting of a rotatable polarizer, power 
meter and spectrometer similar to the one described in $[12,13]$.

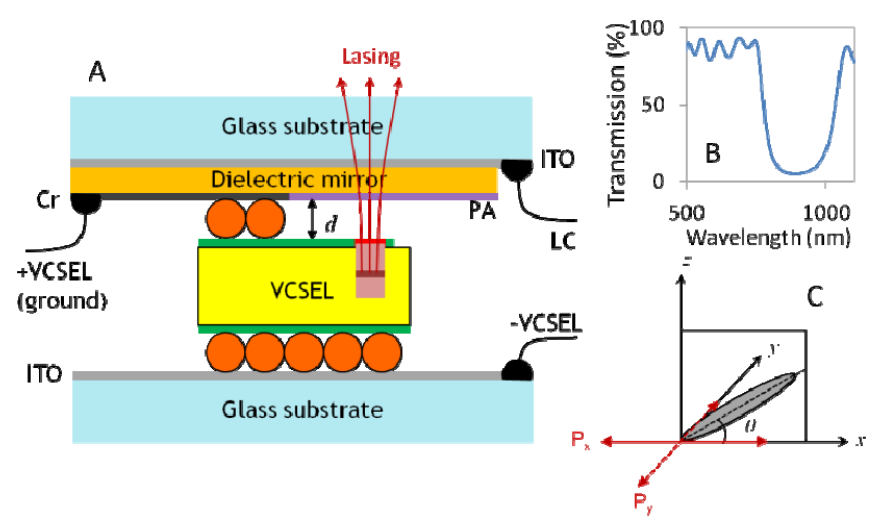

FIG. 1. A: Schematic structure of the LC-VCSEL cell. B: The transmission of the dielectric reflector-coated top glass substrate. C: Coordinate system indicating the tilt of the LC director and the two polarization modes of the VCSEL.

The emission properties of the LC-VCSEL are measured for different ac voltages ( $1 \mathrm{kHz}$ square wave) applied across the LC layer. The optical powers along the two orthogonal directions $\mathrm{x}$ and $\mathrm{y}\left(\mathrm{P}_{\mathrm{x}}\right.$ and $\mathrm{P}_{\mathrm{y}}$ modes $)$ are shown in Fig. 2 as a function of the voltage applied to the LC. The polarization direction changes from $\mathrm{x}$ to $\mathrm{y}$ in voltage zones of $1.1 \sim 1.2 \mathrm{~V}, 1.5 \sim 1.7 \mathrm{~V}$ and $2.1 \sim 2.5 \mathrm{~V}$. The contrast ratio between the two polarizations remains high in the whole voltage range. The switching of the polarization is controllable by applying a suitable voltage over the LC layer.

The polarization switching for different transient voltages is shown in Fig. 3. The optical power of the $\mathrm{P}_{\mathrm{y}}$ mode is measured during the applications of a timedependent voltage signal over the LC. It is observed that when the LC switches dynamically from $0 \mathrm{~V}$ to $3 \mathrm{~V}$ (Fig.3A) the polarization switches between $\mathrm{P}_{\mathrm{x}}$ and $\mathrm{P}_{\mathrm{y}}$ in the same way as for the steady state, shown in Fig.2. We record the switching over $100 \mathrm{~ms}$ which is the typical switching time for this kind of LC, with the given voltage and layer thickness. Figure $3 \mathrm{~A}$ illustrates that full switching of the LC is not needed because the VCSEL switches polarization several times. The actual polarization switching of the VCSEL occurs in a much shorter time than the LC switching time.

Stable and reproducible switching can be achieved by using two voltage amplitudes - just below and just above a polarization switching point - and sufficiently long time intervals to let the LC reach its steady state (Fig.3C). The observed power oscillations of about $100 \mathrm{~Hz}$ come from the data acquisition device. The switching itself occurs in less than $100 \mu \mathrm{s}$ (inset figures in Fig. 3C). Closer analysis of the switching reveals that several jumps between the maximum and minimum level can be distinguished in the $100 \mu$ s interval. This is probably due to fluctuations of the liquid crystal during the reorientation. We believe that the VCSEL switches multiple times between the two polarization states during the switching interval of $100 \mu \mathrm{s}$. The time interval between two polarization switching events can be drastically shortened by using an overdrive scheme which is known from display technology. The voltage is shortly set to a value much higher (or lower) than the steady state value, to speed up the reorientation of the LC director. To demonstrate this principle, we switch the amplitude of the applied voltage from $2.1 \mathrm{~V}$ to $0 \mathrm{~V}$. There is a lag time of about $4 \mathrm{~ms}$ before the laser switches from $\mathrm{P}_{\mathrm{y}}$ to $\mathrm{P}_{\mathrm{x}}$. Almost directly after the switching to $\mathrm{P}_{\mathrm{x}}$ we apply an amplitude of $2.4 \mathrm{~V}$ which results in a lag time of $2 \mathrm{~ms}$ before switching back to $\mathrm{P}_{\mathrm{y}}$ (Fig.3B). After this we apply again the steady state value of $2.1 \mathrm{~V}$. The actual switching time of the laser cannot be detected because the acquisition time of our photodiode is limited to $10 \mu \mathrm{s}$. In this way we demonstrate that with an overdrive scheme one switching cycle can be completed in only a few milliseconds. Note that we use a standard LC in our experiments. Using thinner external cavity or optimized nematic mixtures or novel switching mechanisms based on dual frequency LC or nano-pore polymerized LCs [15] could probably reduce the time of the switching cycle below $100 \mu \mathrm{s}$.

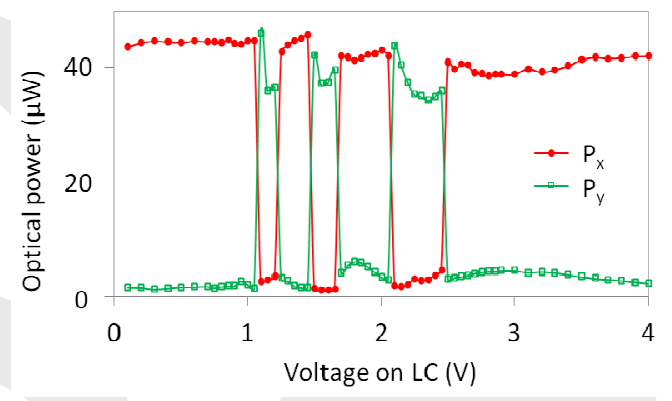

FIG. 2. Measured steady state optical power for two polarizer orientations (along $\mathrm{x}$ and $\mathrm{y}$ axes) at different voltages $(1 \mathrm{kHz})$ applied over the LC layer. The VCSEL current is $1 \mathrm{~mA}$.
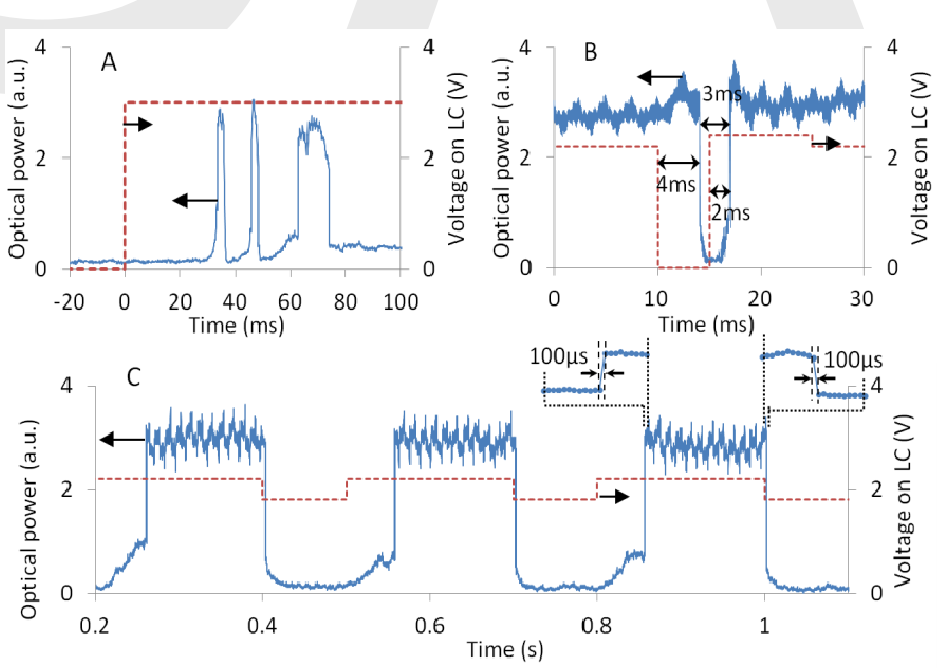

FIG. 3. Transient optical power of the $\mathrm{P}_{\mathrm{y}}$ mode (blue solid line, VCSEL current $1 \mathrm{~mA}$ ) and amplitude of the applied voltage over the LC (red dashed line, $1 \mathrm{kHz}$ ) measured as a function of time. A: LC voltage switching between 0 and $3 \mathrm{~V}$ amplitude. B: fast laser switching by using LC overdrive switching. C: periodic switching between two voltage amplitudes, with inset of the switching. 
The emission wavelength of the two polarization modes $\left(\mathrm{P}_{\mathrm{x}}\right.$ and $\left.\mathrm{P}_{\mathrm{y}}\right)$ as a function of the voltage over the LC is shown in Fig. 4. It is observed that the emission wavelength of polarization $\mathrm{P}_{\mathrm{x}}$ continuously decreases over $0.5 \mathrm{~nm}$ with increasing voltage and that after the switch to $\mathrm{P}_{\mathrm{y}}$ the lasing wavelength is about $1 \mathrm{~nm}$ smaller. This switch to $\mathrm{P}_{\mathrm{y}}$ is observed in the intervals $1 \sim 1.1 \mathrm{~V}$, $1.5 \sim 1.8 \mathrm{~V}$ or $2.2 \sim 2.6 \mathrm{~V}$. Compared to the polarization measurement in Fig.2, there is a small mismatch of the voltage range where the switches happen, which is due the fact that the measurements are not exactly reproduced at different times. The optical power versus VCSEL current at different voltages applied over the LC layer has also been measured (not shown). The threshold current is calculated using a least square linear regression for the linear part of the power versus current (P-I) curve [13]: the intersection with the current-axis yields the threshold current. It is observed that the threshold current reaches a local maximum at the voltage values where the polarization switches.

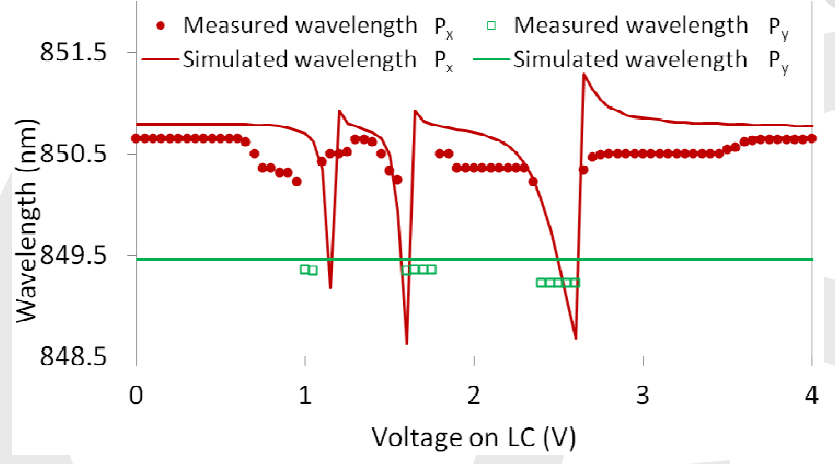

FIG. 4. Measured emission wavelength (red dots for $\mathrm{P}_{\mathrm{x}}$; green squares for $\mathrm{P}_{\mathrm{y}}$ ) and simulated emission wavelength (red line for $\mathrm{P}_{\mathrm{x}}$; green line for $\mathrm{P}_{\mathrm{y}}$ ) as a function of the voltage amplitude over the LC layer. The VCSEL current is $1 \mathrm{~mA}$, the frequency of the $\mathrm{LC}$ is $1 \mathrm{kHz}$.

To understand the observed behaviors of the LCVCSEL, we have done theoretical analysis. The simulations of the VCSEL with external cavity are carried out using a plane-wave expansion method [16] based on the transfer matrix method [17]. The liquid crystal configuration is described by the tilt angle distribution $\theta(\mathrm{z})$ of the director (indicating the average orientation of the LC molecules, see Fig. 1C). This distribution is calculated with a simple equation based on the minimization of the Oseen-Frank free energy [18]. The $\theta(\mathrm{z})$ steady-state distribution for different applied voltages over the LC layer is shown in Fig.5. The round trip gain in the combined VCSEL/external cavity is calculated as a function of the wavelength. The threshold for lasing is obtained by increasing the imaginary part of refractive index of the active layer $n^{i m}$ until the round trip gain reaches 1 for a certain wavelength, the resonance wavelength $\lambda_{\text {res. }}$ The threshold gain is calculated from $G_{t h}=4 \pi / \lambda_{\text {res }} n^{i m}$. The parameters used in the simulation are listed in Table 1 . The simulation results for lasing wavelength and threshold gain for two polarizations as a function of the voltage applied over the LC are shown in Fig. 4 and Fig. 6.

Table 1. LC-VCSEL parameters

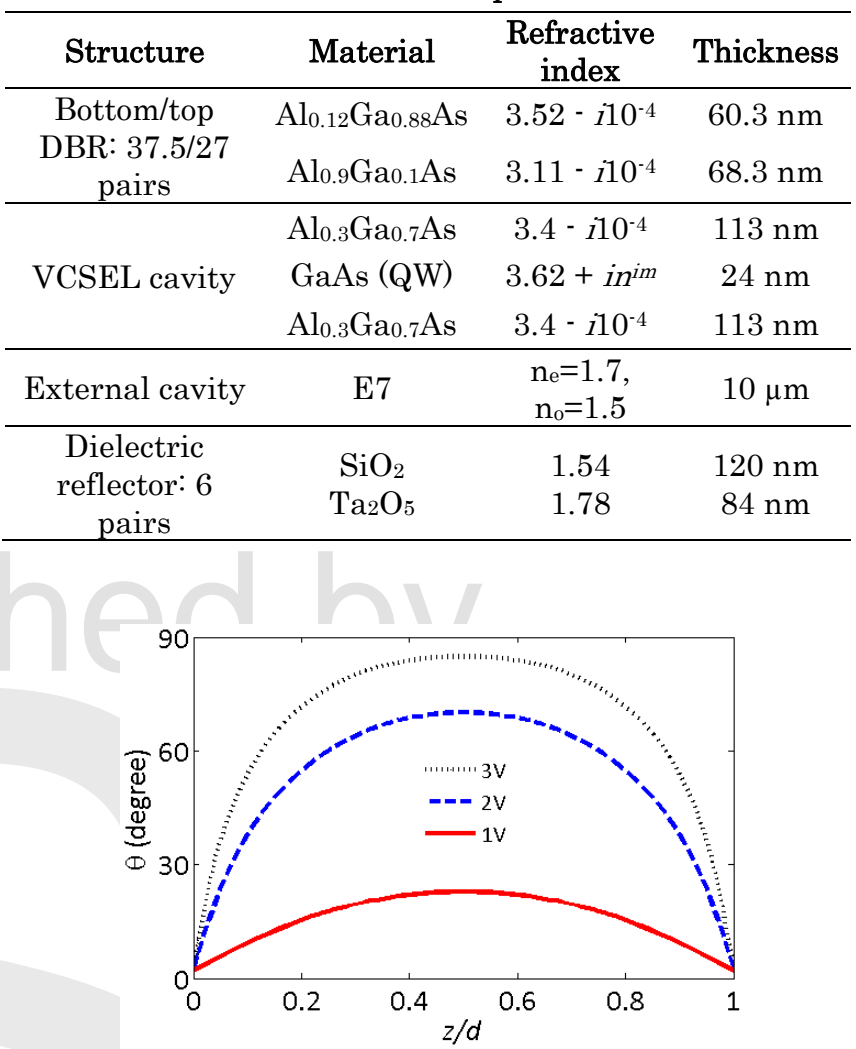

FIG. 5. Simulated tilt angle distribution $\theta(\mathrm{z})$ for three different voltages.

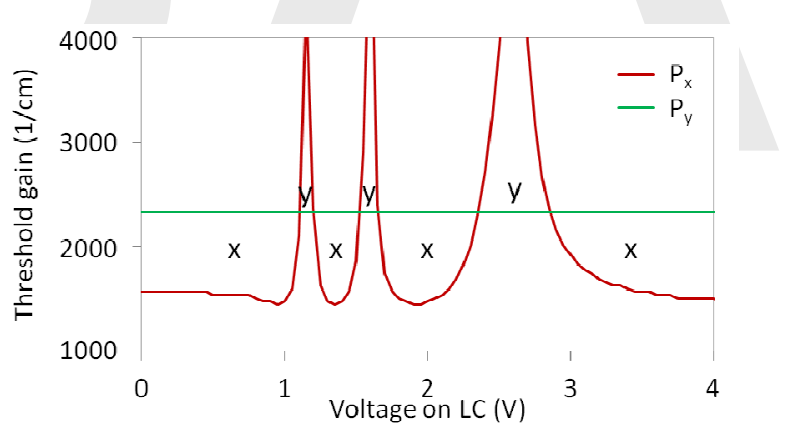

FIG. 6. Simulated threshold gain (red line for $\mathrm{P}_{\mathrm{x}}$; green line for $\mathrm{P}_{\mathrm{y}}$ ), as a function of the voltage over the LC layer. The polarization mode with the lower threshold gain is indicated by its orientation ( $\mathrm{x}$ or $\mathrm{y}$ ) for different voltage zones.

The measurements of wavelength and threshold current agree well with the simulation of the emission wavelength (Fig.4) and threshold gain (Fig.6), respectively. The periodic changes observed in the measurements can be explained as follows. The LC reorientation modulates the refractive index for $\mathrm{x}^{-}$ polarized light and the optical path length for the mode $P_{x}$ (see also [17]). The polarization mode $\mathrm{P}_{\mathrm{y}}$ always encounters the ordinary refractive index and its optical path length is fixed. As a result, the reflection and the 
lasing threshold for $\mathrm{P}_{\mathrm{x}}$ change with the applied voltage, while the values for $\mathrm{P}_{\mathrm{y}}$ remain constant (shown in Fig.6). When the lasing threshold for $\mathrm{P}_{\mathrm{x}}$ increases above that for $\mathrm{P}_{\mathrm{y}}$, the polarization switches from $\mathrm{P}_{\mathrm{x}}$ to $\mathrm{P}_{\mathrm{y}}$ (shown in Fig.2) and the threshold current reaches a local maximum.

In conclusion, a tunable external cavity for a VCSEL is realized by adding a dielectric reflector in front of the exit window and filling the resulting space with nematic LC. By applying a voltage over the LC layer, the LC director reorients and the optical path length in the external cavity is tuned. The polarization state and emission wavelength of the resulting LC-VCSEL can be controlled by the voltage applied to the LC layer. Using current state-of-the-art LC-VCSEL technology, we believe that the switching cycles can be brought down to less than $100 \mu$ s with nanosecond polarization switching.

This work was carried out in the framework of the IAP project Photonics@be funded by the Belgian Science Policy and the FWO project under grant G.0656.09N. K. Panajotov is grateful to the Methusalem foundation for financial support.
14. K. Panajotov, Y. Xie, M. Dems, C. Belmonte, H. Thienpont, J. beeckman and K. Neyts, Laser Phys. Lett. 10 (10), 105003 (2013).

15. M. Mohammadimasoudi, J. Beeckman, J. Shin, K. Lee and K. Neyts, Opt. Express 22 (16), 19098-19107 (2014).

16. L. Penninck, J. Beeckman, P. D. Visschere and K. Neyts, Phys. Rev. E 85, 041702 (2012).

17. K. Panajotov and H. Thienpont, Opt. Express 19 (18), 16749 (2011).

18. T. Scharf, Polarized light in liquid crystals and polymers. (Wiley Interscience, United State of America, 2007).

\section{References}

1. Y. G. Zhao and J. G. McInerney, IEEE J. Quantum. Elect. 32 (11), 1950-1958 (1996).

2. C. Degen, I. Fischer and W. Elsäßer, Opt. Express 5 (3), 38-47 (1999).

3. J. Dellunde, A. Valle and K. A. Shore, J. Opt. Soc. Am. B 13 (11), 2477-2783 (1996).

4. A. Valle, L. Pesquera and K. A. Shore, IEEE Photonic. Tech. L. 10 (5) (1998).

5. T. H. Russell and T. D. Milster, Appl. Phys. Lett. 70, 2520-2522 (1997).

6. K. Panajotov, M. Arizaleta, M. Camarena, H. Thienpont, H. J. Unold, J.-M. Ostermann, R. Michalzik, Appl. Phys. Lett. 84 (15), 2763-2765 (2004).

7. Q. Wang, B. Guan, K. Liu, X. Liu, X. Jiang, Y. Ma, S. Arafi and G. Shen, Opt. Laser Techl. 63, 19-23 (2014).

8. A. V. Barve, Y. Zheng, L. A. Johansson, A. Mehta, A. Husain and L. A. Coldren, Opt. Express 21 (25), 31092-31097 (2013).

9. P. Perez, A. Valle, L. Pesquera and A. Quirce, IEEE J. Sel. Top. Quant. 19 (4), 1700408 (2013).

10. A. A. Qader, Y. Hong and K. A. Shore, IEEE Photonic. Tech. L. 25 (12), 1173-1176 (2013).

11. C. Levallois, B. Caillaud, J. L. D. de la Tocnaye, L. Dupont, A. Le Corre, H. Folliot, O. Dehaese and S. Loualiche, Appl. Phys. Lett. 89 (1), 011102 (2006).

12. Y. Xie, J. Beeckman, W. Woestenborghs, K. Panajotov and K. Neyts, IEEE Photonic. Tech. L. 24 (17), 1509-1512 (2012).

13. Y. Xie, J. Beeckman, K. Panajotov and K. Neyts, IEEE Photonics J. 6 (1), 1500010 (2014). 


\section{Full references}

1. Y. G. Zhao and J. G. McInerney, "TransverseMode Control of Vertical-Cavity SurfaceEmitting Lasers," IEEE J. Quantum. Elect. 32 (11), 1950-1958 (1996).

2. C. Degen, I. Fischer and W. Elsäßer, "Transverse modes in oxide confined VCSELs: Influence of pump profile, spatial hole burning, and thermal effects," Opt. Express 5 (3), 38-47 (1999).

3. J. Dellunde, A. Valle and K. A. Shore, "Transverse mode selection in external cavity vertical cavity surface emitting laser diodes," J. Opt. Soc. Am. B 13 (11), 2477-2783 (1996).

4. A. Valle, L. Pesquera and K. A. Shore, "Polarization Selection and Sensitivity of External Cavity Vertical-Cavity SurfaceEmitting Laser Diodes," IEEE Photonic. Tech. L. 10 (5) (1998).

5. T. H. Russell and T. D. Milster, "Polarization switching control in vertical-cavity surfaceemitting lasers,” Appl. Phys. Lett. 70, 2520-2522 (1997).

6. K. Panajotov, M. Arizaleta, M. Camarena, H. Thienpont, H. J. Unold, J.-M. Ostermann, R. Michalzik, "Polarization switching induced by phase change in extremely short external cavity vertical-cavity surface-emitting lasers," Appl. Phys. Lett. 84 (15), 2763-2765 (2004).

7. Q. Wang, B. Guan, K. Liu, X. Liu, X. Jiang, Y. $\mathrm{Ma}$, S. Arafi and G. Shen, "Temperature dependent polarization switch of $850-\mathrm{nm}$ VCSELs with different apertures," Opt. Laser Techl. 63, 19-23 (2014).

8. A. V. Barve, Y. Zheng, L. A. Johansson, A. Mehta, A. Husain and L. A. Coldren, "Fast, electrically controlled polarization modulation of multimode vertical-cavity surface-emitting lasers by RF frequency modulation," Opt. Express 21 (25), 31092-31097 (2013).

9. P. Perez, A. Valle, L. Pesquera and A. Quirce, "All-Optical Inverter Based on Polarization Switching in VCSELs Subject to Single and Dual Optical Injection" IEEE J. Sel. Top. Quant. 19 (4), 1700408 (2013).

10. A. A. Qader, Y. Hong and K. A. Shore, "Robust Irreversible Polarization Switching in Optically Injected VCSELs" IEEE Photonic. Tech. L. 25 (12), 1173-1176 (2013).

11. C. Levallois, B. Caillaud, J. L. D. de la Tocnaye, L. Dupont, A. Le Corre, H. Folliot, O. Dehaese and S. Loualiche, "Long-wavelength verticalcavity surface-emitting laser using an electrooptic index modulator with $10 \mathrm{~nm}$ tuning range," Appl. Phys. Lett. 89 (1), 011102 (2006).
12. Y. Xie, J. Beeckman, W. Woestenborghs, K. Panajotov and K. Neyts, "VCSEL with photoaligned liquid crystal overlay," IEEE Photonic. Tech. L. 24 (17), 1509-1512 (2012).

13. Y. Xie, J. Beeckman, K. Panajotov and K. Neyts, "Vertical-cavity surface-emitting laser with a chiral nematic liquid crystal overlay," IEEE Photonics J. 6 (1), 1500010 (2014).

14. K. Panajotov, Y. Xie, M. Dems, C. Belmonte, H. Thienpont, J. beeckman and K. Neyts, "Verticalcavity surface-emitting laser emitting circularly polarized light," Laser Phys. Lett. 10 (10), 105003 (2013).

15. M. Mohammadimasoudi, J. Beeckman, J. Shin, K. Lee and K. Neyts, "Widely tunable chiral nematic liquid crystal optical filter with microsecond switching time," Opt. Express 22 (16), 19098-19107 (2014).

16. L. Penninck, J. Beeckman, P. D. Visschere and K. Neyts, "Light emission from dye-doped cholesteric liquid crystals at oblique angles: Simulation and experiment," Phys. Rev. E 85, 041702 (2012).

17. K. Panajotov and H. Thienpont, "Vertical-cavity surface-emitting laser with liquid crystal overlay," Opt. Express 19 (18), 16749 (2011).

18. T. Scharf, Polarized light in liquid crystals and polymers. (Wiley Interscience, United State of America, 2007). 